\title{
Metabolite profiling of blood from individuals undergoing planned myocardial infarction reveals early markers of myocardial injury
}

\author{
Gregory D. Lewis, 1,2,3,4 Ru Wei, ${ }^{4}$ Emerson Liu, 1,2,3 Elaine Yang, ${ }^{4}$ Xu Shi, ${ }^{2}$ Maryann Martinovic, ${ }^{1}$ \\ Laurie Farrell, ${ }^{1}$ Aarti Asnani, ${ }^{1,2,4}$ Marcoli Cyrille, ${ }^{2}$ Arvind Ramanathan, ${ }^{4}$ Oded Shaham, 4,5 \\ Gabriel Berriz, ${ }^{6}$ Patricia A. Lowry, ${ }^{1}$ Igor F. Palacios, ${ }^{1}$ Murat Taşan, ${ }^{6}$ Frederick P. Roth, ${ }^{6}$ \\ Jiangyong Min, ${ }^{7}$ Christian Baumgartner, ${ }^{8}$ Hasmik Keshishian, ${ }^{4}$ Terri Addona, ${ }^{4}$ \\ Vamsi K. Mootha, ${ }^{4,5}$ Anthony Rosenzweig,, ${ }^{7}$ Steven A. Carr, ${ }^{4}$ Michael A. Fifer, ${ }^{1}$ \\ Marc S. Sabatine, 1,3,9 and Robert E. Gerszten ${ }^{1,2,3,4}$

\begin{abstract}
${ }^{1}$ Cardiology Division and ${ }^{2}$ Center for Immunology and Inflammatory Diseases, Massachusetts General Hospital, Boston, Massachusetts, USA ${ }^{3}$ Donald W. Reynolds Cardiovascular Clinical Research Center on Atherosclerosis at Harvard Medical School, Boston, Massachusetts, USA. Massachusetts, USA. ${ }^{6}$ Department of Biological Chemistry and Molecular Pharmacology, Harvard Medical School, Boston, Massachusetts, USA. ${ }^{7}$ Cardiovascular Institute, Beth Israel Deaconess Medical Center, Boston, Massachusetts, USA. ${ }^{8}$ Research Group for Clinical Bioinformatics, University for Health Sciences, Medical Informatics and Technology (UMIT), Tirol, Austria. ${ }^{9}$ Cardiovascular Division,
\end{abstract} \\ ${ }^{4}$ Broad Institute of MIT and Harvard, Cambridge, Massachusetts, USA. ${ }^{5}$ Center for Human Genetic Research, Massachusetts General Hospital, Boston,
} Brigham and Women's Hospital, Boston, Massachusetts, USA.

\begin{abstract}
Emerging metabolomic tools have created the opportunity to establish metabolic signatures of myocardial injury. We applied a mass spectrometry-based metabolite profiling platform to 36 patients undergoing alcohol septal ablation treatment for hypertrophic obstructive cardiomyopathy, a human model of planned myocardial infarction (PMI). Serial blood samples were obtained before and at various intervals after PMI, with patients undergoing elective diagnostic coronary angiography and patients with spontaneous myocardial infarction (SMI) serving as negative and positive controls, respectively. We identified changes in circulating levels of metabolites participating in pyrimidine metabolism, the tricarboxylic acid cycle and its upstream contributors, and the pentose phosphate pathway. Alterations in levels of multiple metabolites were detected as early as 10 minutes after PMI in an initial derivation group and were validated in a second, independent group of PMI patients. A PMI-derived metabolic signature consisting of aconitic acid, hypoxanthine, trimethylamine $\mathrm{N}$-oxide, and threonine differentiated patients with SMI from those undergoing diagnostic coronary angiography with high accuracy, and coronary sinus sampling distinguished cardiac-derived from peripheral metabolic changes. Our results identify a role for metabolic profiling in the early detection of myocardial injury and suggest that similar approaches may be used for detection or prediction of other disease states.
\end{abstract}

\section{Introduction}

Novel metabolomics technologies have enhanced the feasibility of acquiring high-throughput snapshots of a whole organism's metabolic status. Metabolomics studies to date have focused principally on model organisms (1-4). However, the profiling of low-molecular weight biochemicals, including lipids, sugars, and amino acids, that serve as substrates and products in metabolic pathways is particularly relevant to human conditions such as myocardial ischemia (5). In addition to serving as disease biomarkers, circulating metabolites may themselves participate in previously unanticipated roles as regulatory signals with hormone-like functions. In this regard, a recent publication suggests that the tricarboxylic acid (TCA) cycle intermediate succinate, released from ischemic tissues, may activate a novel $G$ protein-coupled receptor in the kidney to modulate blood pressure (6).

Nonstandard abbreviations used: CK, creatine kinase; CK-MB, CK muscle and brain subunits; HOCM, hypertrophic obstructive cardiomyopathy; LC, liquid chromatography; MI, myocardial infarction; MS, mass spectrometry; PMI, planned MI; SMI, spontaneous MI; TCA, tricarboxylic acid; TMNO, trimethylamine N-oxide.

Conflict of interest: While this manuscript was under review, the Massachusetts General Hospital filed for patents related to metabolic biomarkers of myocardial ischemia on behalf of R.E. Gerszten, M.A. Fifer, and M.S. Sabatine.

Citation for this article: J. Clin. Invest. 118:3503-3512 (2008). doi:10.1172/JCI35111.
However, metabolomics techniques still suffer from signal-tonoise issues, and applications to humans have been limited by the profound degree of interindividual variability. Studies to identify novel disease-related pathways have been further restricted by the inherent unpredictability of the onset of pathological states. Human metabolomics studies have also been complicated by potentially confounding clinical variables such as diet or drug effects, particularly if NMR or mass spectrometry-based (MS-based) profiling techniques are used without unambiguously identifying metabolites associated with peaks of interest. Indeed, published findings of ${ }^{1} \mathrm{H}-\mathrm{NMR}$ spectral variation correlating with coronary artery disease severity (7) were later found to be confounded by cholesterol-lowering (i.e., HMG-CoA reductase inhibition) therapy (8). The analysis of samples from large patient cohorts, stratified by known risk factors or exposures, may minimize the impact of clinical confounding variables (8). However, the throughput of many technologies, particularly those that are MS based, currently precludes the analysis of large numbers of samples.

To help circumvent these problems, we applied a targeted MS-based metabolomics platform, which provides high analyte specificity $(9,10)$, to serial blood samples obtained from carefully phenotyped patients undergoing alcohol septal ablation for hypertrophic obstructive cardiomyopathy (HOCM). This human 


\section{Table 1}

Baseline clinical characteristics of study cohorts

\begin{tabular}{|c|c|c|c|c|c|}
\hline & $\begin{array}{l}\text { PMI derivation } \\
\qquad(n=20)\end{array}$ & $\begin{array}{l}\text { PMI validation } \\
\qquad(n=16)\end{array}$ & $\begin{array}{l}\text { PMI control } \\
\quad(n=16)\end{array}$ & $\begin{array}{c}\text { SMI } \\
(n=12)\end{array}$ & $\begin{array}{l}\text { SMI control } \\
\quad(n=9)\end{array}$ \\
\hline $\begin{array}{l}\text { Age (yr) } \\
\text { Male }(\%) \\
\text { White }(\%)\end{array}$ & $\begin{array}{c}63 \pm 14 \\
40 \\
90\end{array}$ & $\begin{array}{c}60 \pm 15 \\
50 \\
94\end{array}$ & $\begin{array}{c}63 \pm 13 \\
63 \\
83\end{array}$ & $\begin{array}{c}61 \pm 12 \\
75 \\
83\end{array}$ & $\begin{array}{c}64 \pm 13 \\
78 \\
78\end{array}$ \\
\hline $\begin{array}{l}\text { Creatinine baseline }(\mathrm{mg} / \mathrm{dl}) \\
\text { Peak troponin T (ng/ml) } \\
\text { Peak CK }(\mathrm{U} / \mathrm{l}) \\
\text { Peak CK-MB }(\mathrm{ng} / \mathrm{ml}) \\
\text { Total cholesterol }(\mathrm{mg} / \mathrm{dl})\end{array}$ & $\begin{aligned} 0.9 & \pm 0.2 \\
5.0 & \pm 3.0 \\
1,149 & \pm 509 \\
187 & \pm 98 \\
167 & \pm 26\end{aligned}$ & $\begin{aligned} 1.0 & \pm 0.2 \\
4.0 & \pm 2.0 \\
1,296 & \pm 1,328 \\
217 & \pm 102 \\
172 & \pm 48\end{aligned}$ & $\begin{aligned} & 1.1 \pm 0.3 \\
&<0.01^{A} \\
& 100 \pm 25^{A} \\
& 3 \pm 0.7^{A} \\
& 168 \pm 64\end{aligned}$ & $\begin{aligned} 1.1 & \pm 0.3 \\
8.8 & \pm 4.5^{B} \\
2,929 & \pm 383^{B} \\
324 & \pm 138^{B} \\
183 & \pm 38\end{aligned}$ & $\begin{array}{l}1.1 \pm 0.2 \\
N A \\
101 \pm 25^{A} \\
2.6 \pm 0.7^{A} \\
172 \pm 30\end{array}$ \\
\hline $\begin{array}{l}\text { Aspirin exposure }(\%) \\
\text { Beta-blocker exposure (\%) } \\
\text { Calcium channel blocker exposure (\%) } \\
\text { Statin exposure (\%) } \\
\text { Unfractionated heparin exposure (\%) }\end{array}$ & $\begin{array}{c}100 \\
90 \\
40 \\
50 \\
100\end{array}$ & $\begin{array}{c}100 \\
70 \\
30 \\
44 \\
100\end{array}$ & $\begin{array}{l}100 \\
81 \\
10 \\
75 \\
70\end{array}$ & $\begin{array}{c}100 \\
92 \\
17 \\
25 \\
100\end{array}$ & $\begin{array}{c}100 \\
100 \\
56 \\
56 \\
100\end{array}$ \\
\hline
\end{tabular}

Continuous variables are mean $\pm \mathrm{SD}$; categorical variables are shown as percentages. ${ }^{\mathrm{A} P}<0.05$ versus $\mathrm{PMI}$ derivation, $\mathrm{PMI}$ validation, and SMI cohorts. $\mathrm{B} P<0.05$ versus all PMI and SMI control cohorts.

model of planned myocardial infarction (PMI; refs. 11, 12) faithfully reproduces spontaneous myocardial infarction (SMI; refs. 13-15). The serial sampling before and after the controlled myocardial insult allows each patient to serve as his or her own biological control. To assess whether metabolic alterations were localized to the heart after myocardial injury, we simultaneously sampled the venous effluent from the heart as well as peripheral blood. The specificity of metabolic alterations was also verified by studying patients undergoing catheterization without the induction of myocardial infarction (MI). Finally, to assess generalizability, we tested our metabolic biomarkers in patients with SMI.

\section{Results}

PMI recapitulates SMI. Clinical characteristics of the HOCM patients and other study populations are detailed in Table 1 . The septal ablation recapitulated important clinical features of SMI, including substernal chest pain and electrocardiographic changes as well as development of echocardiographic evidence of septal wall motion abnormalities, as previously described by our group and others (13-15). The standard biochemical metrics of myocardial injury, creatine kinase (CK) muscle and brain subunits (CK-MB) and troponin T, were within normal limits prior to septal ablation and increased to $200 \pm 98 \mathrm{ng} / \mathrm{ml}$ and $4.5 \pm 2.6 \mathrm{ng} / \mathrm{ml}$, respectively. CK-MB peaked at $8.9 \pm 4.5$ hours and cardiac troponin $\mathrm{T}$ at $14.9 \pm 8.0$ hours after PMI, time courses consistent with SMI (16).

Derivation and validation of early metabolic changes in peripheral plasma. We first studied peripheral blood samples across the range of time points available (10 minutes to 24 hours) to characterize metabolic alterations associated with PMI in a derivation cohort of 20 patients. Table 2 describes the most significantly changed metabolites 10 minutes after the onset of myocardial injury. We observed a total of 7 metabolites that were significantly changed (nominal $P<0.005$ ), with an estimated false discovery rate of approximately $11 \%$ (see Methods). Based on this false discovery rate, we expected that 6 of the 7 metabolites were truly differentially changed.

Because biomarker discovery studies are vulnerable to unintentional overfitting of data $(17,18)$, we then assessed the performance of metabolites that were significantly changed in the derivation cohort in an independent validation cohort of patients also undergoing the septal ablation procedure $(n=16$; Table 2$)$. Significant changes $(P<0.05)$ were noted in the validation cohort for 6 of the 7 metabolites observed in the derivation cohort. These metabolites all showed concordance with the derivation cohort in the direction of change, and the magnitude of change in the 2 cohorts was highly correlated $\left(r^{2}=0.87, P=0.01\right)$. Metabolic changes included products of purine and pyrimidine catabolism (hypoxanthine and malonic acid, respectively) as well as several amino acids. Of note, the alterations in these metabolites were seen when no significant rises in the clinically available biomarkers, $\mathrm{CK}-\mathrm{MB}$ and troponin $\mathrm{T}$, were detectable in the plasma ( $P=$ NS for both).

By 60 minutes after PMI, more metabolic changes were noted (Table 3). All of the metabolic changes documented at 10 minutes were also observed at 60 minutes, underscoring the consistency of our findings. At 60 minutes, 13 metabolites were significantly changed (nominal $P<0.005$ ), with an estimated false discovery rate of $6.5 \%$. Significant changes in 10 of the 13 metabolites observed in the derivation cohort were noted in the validation cohort as well $(P<0.05)$, with strong trends for the remaining 3 metabolites. Again, the magnitude of change in the 2 cohorts was highly correlated at this time point $\left(r^{2}=0.93, P<0.0001\right)$. By 60 minutes, additional changes in purine metabolites (xanthine and inosine), an inflammatory mediator (methylhistamine), and other amines and amino acids were detected.

We explored the specificity of the findings observed in the PMI cohort by examining blood samples from patients undergoing routine cardiac catheterization, without the induction of MI that occurs in the unique ablation injury model. Whereas the metabolic changes in the derivation and validation cohorts were highly correlated, as noted above, there was no correlation between the derivation cohort and the catheterization control group overall ( $P=0.47$ at 10 minutes; $P=0.76$ at 60 minutes). However, cardiac catheterization alone was associated with changes in 3 metabolites, tryptophan, tyrosine, and phenylalanine, at either the 10-minute or the 60 -minute time point $(P<0.01)$. These 3 metabolites were therefore not included in Tables 2 and 3 and were excluded from further analysis. Thus, metabolites with changes that were not spe- 
Table 2

Metabolite changes in the peripheral blood 10 minutes after myocardial injury

\begin{tabular}{|c|c|c|c|c|}
\hline \multirow[t]{2}{*}{ Metabolite } & \multicolumn{2}{|c|}{ Derivation cohort } & \multicolumn{2}{|c|}{ Validation cohort } \\
\hline & Change & $P$ & Change & $P$ \\
\hline Alanine & $-18.2(-23.5$ to -13.6$)$ & 0.0003 & $-12.4(-18.5$ to -8.2$)$ & 0.001 \\
\hline Aminoisobutyric acid & $31.7(13.4$ to 46.9$)$ & 0.0031 & $2.9(-20.8$ to 32.5$)$ & 0.98 \\
\hline Hypoxanthine & 189.6 (88.9 to 408.4$)$ & 0.0005 & 249.6 (105.1 to 335.1 ) & 0.0006 \\
\hline Isoleucine/leucine & $7.0(3.6$ to 17.0$)$ & 0.0016 & $7.4(0.8$ to 16.9$)$ & 0.048 \\
\hline Malonic acid & 16.0 (12.4 to 93.0) & 0.0019 & 24.2 (19.7 to 57.4$)$ & 0.001 \\
\hline Threonine & $-10.0(-11.9$ to -5.2$)$ & 0.0036 & $-4.9(-11.3$ to 0.3$)$ & 0.041 \\
\hline TMNO & $-15.5(-29.0$ to -8.9$)$ & 0.0031 & $-24.4(-33.7$ to -7.3$)$ & 0.026 \\
\hline
\end{tabular}

Metabolites with $P<0.005$ in the derivation cohort are shown, listed in alphabetical order. Change values denote median percent change versus baseline; values in parentheses represent interquartile range. Metabolites of identical retention times - and parent-daughter ion pairs (e.g., isoleucine and leucine) - cannot be distinguished by the platform. increased in the peripheral samples also increased in the coronary sinus samples, underscoring the consistency of our findings. Of the 7 metabolites that increased in the peripheral blood, 5 showed at least a 1.3-fold enrichment in the coronary sinus at 10 minutes after myocardial injury. As expected, a transmyocardial enrichment pattern was evident for metabolites related to myocardial anaerobic metabolism, including lactic acid and succinic acid, as well as ATP degradation products, such as hypoxanthine and AMP. Furthermore, changes in the levels of certain metabolites were first apparent in the coronary sinus, and were only later detected in the peripheral samples. For example, inosine and 1-methylhistamine levels were significantly cific to myocardial injury and that may instead reflect procedural events such as arteriotomy or catheter manipulation were eliminated using the appropriate patient controls.

Kinetic analysis of metabolic changes in peripheral plasma. Figure 1 shows representative metabolites across a spectrum of time points after PMI. Some metabolic changes were relatively transient $(\leq 120$ minutes), as seen with malonic acid and alanine, whereas other early metabolic changes persisted ( $\geq 240$ minutes), as seen with hypoxanthine, aconitic acid, trimethylamine N-oxide (TMNO), and threonine. Later-appearing metabolites included anthranilic acid and creatine, the latter of which was detected in a time course in our study consistent with that of CK-MB release from cardiomyocytes undergoing necrosis (19).

Early metabolic changes in coronary sinus plasma. We further examined the specificity of our findings by exploring the anatomic origin of the early metabolic changes. In a subgroup of 13 patients, we compared metabolite levels in samples obtained simultaneously from the peripheral blood and from a catheter placed in the coronary sinus, the venous outflow of the heart. This simultaneous sampling allowed us to identify transmyocardial enrichment of metabolites. As a reference for comparison, B-type natriuretic peptide, a protein released from the heart in response to left ventricular wall stress, was enriched approximately 1.3 -fold in the coronary sinus samples 60 minutes after injury (data not shown). We assessed whether the metabolites that increased at 10 or $60 \mathrm{~min}$ utes in the peripheral blood (1-methylhistamine, aminoisobutyric acid, hypoxanthine, inosine, isoleucine/leucine, malonic acid, and xanthine; Tables 2 and 3) were also enriched in the coronary sinus at 10 minutes (Table 4). We evaluated relative changes in metabolite levels in the coronary sinus compared with the periphery for the rest of the metabolites in our platform as well. Additional biomarkers that showed greater than 1.3-fold transmyocardial enrichment 10 minutes after PMI are shown in Table 5. Peripheral and coronary data for the 60-minute time point are shown in Tables 6 and 7. All of the metabolites that

\section{Table 3}

Metabolite changes in the peripheral blood 60 minutes after myocardial injury

\begin{tabular}{lcccc} 
Metabolite & \multicolumn{2}{c}{$\begin{array}{c}\text { Derivation cohort } \\
\text { Change }\end{array}$} & $\boldsymbol{P}$ & \multicolumn{2}{c}{$\begin{array}{c}\text { Validation cohort } \\
\text { Change }\end{array}$} & $\boldsymbol{P}$ \\
1-Methylhistamine & $12.9(3.2$ to 23.0$)$ & 0.004 & $33.2(-1.9$ to 67.0$)$ & 0.028 \\
Alanine & $-22.6(-24.8$ to -20.2$)$ & 0.0002 & $-11.9(-28.6$ to -7.1$)$ & 0.012 \\
Aminoisobutyric acid & $45.5(24.9$ to 65.3$)$ & 0.0012 & $27.7(0.9$ to 43.0$)$ & 0.071 \\
Choline & $-9.4(-15.2$ to -1.1$)$ & 0.0025 & $-10.3(-14.2$ to 1.7$)$ & 0.049 \\
Hypoxanthine & $301.7(136.7$ to 536.1$)$ & 0.0003 & $342.5(201.4$ to 390.7$)$ & 0.0076 \\
Isoleucine/leucine & $10.6(2.1$ to 18.6$)$ & 0.0017 & $13.3(3.2$ to 17.2$)$ & 0.049 \\
Inosine & $51.6(7.2$ to184.0) & 0.0011 & $35.7(-24.4$ to 145.0$)$ & 0.110 \\
Malonic acid & $47.9(5.9$ to 96.2$)$ & 0.0022 & $43.7(16.9$ to 86.0$)$ & 0.003 \\
Proline & $-4.6(-9.1$ to -2.8$)$ & 0.0036 & $-4.2(-15.4$ to 2.8$)$ & 0.099 \\
Serine & $-10.0(-18.8$ to -4.8$)$ & 0.0015 & $-8.9(-19.4$ to -0.6$)$ & 0.034 \\
Threonine & $-7.5(-14.4$ to -3.1$)$ & 0.0003 & $-6.9(-13.3$ to -2.8$)$ & 0.0037 \\
TMN0 & $-20.2(-27.2$ to -9.6$)$ & 0.0007 & $-32.4(-39.1$ to -17.7$)$ & 0.028 \\
Xanthine & $48.1(18.7$ to 82.9$)$ & 0.0027 & $71.9(26.9$ to 119.3$)$ & 0.004
\end{tabular}

Metabolites are listed in alphabetical order. Change values denote median percent change versus baseline; values in parentheses represent interquartile range. Metabolites of identical retention times - and parent-daughter ion pairs (e.g., isoleucine and leucine) - cannot be distinguished by the platform. 


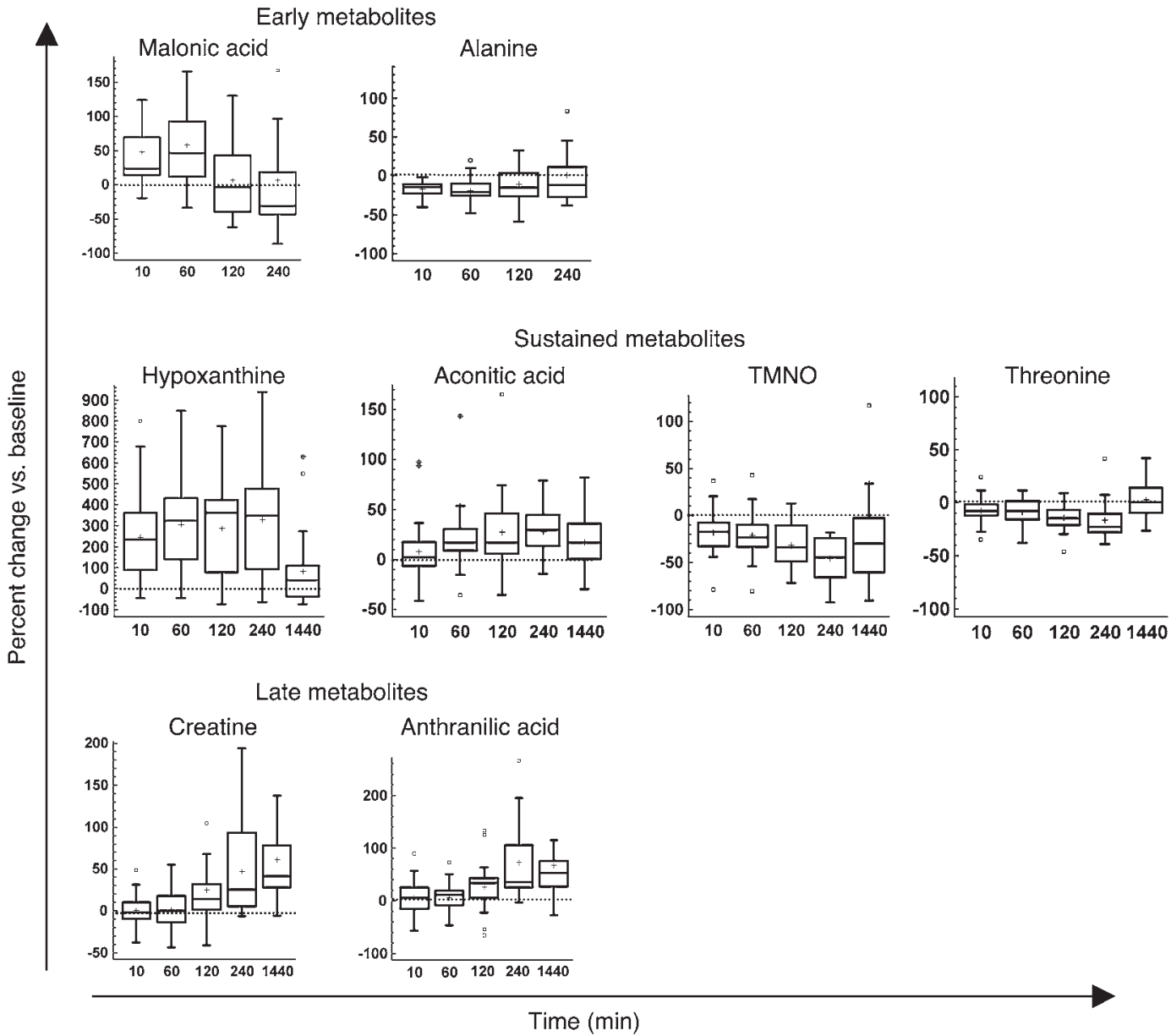

Figure 1

Kinetics of metabolic changes in peripheral human plasma after planned myocardial injury. Representative metabolites demonstrating transient ( $\leq 120$ minutes after injury; see Table 2), sustained ( $\geq 240$ minutes after injury), and late (1,440 minutes after injury, i.e., 1 day) changes in plasma levels compared with baseline are shown. Data represent the entire cohort of 36 patients: boxes denote interquartile range, lines denote median, "+" denotes mean, whiskers denote fifth and ninety-fifth percentiles, and outliers are shown by points.

minutes), we focused on the group of metabolites that changed significantly in the peripheral blood in a sustained pattern after PMI (Figure 1). These 4 metabolites in the platform - hypoxanthine, aconitic acid, TMNO, and threonine - were significantly changed at each of the 60-, 120-, and 240-minute time points in the entire PMI cohort ( $P<0.05$ for each). The median changes across these 3 time points compared with baseline are shown in Figure 3A. We next examined the difference in levels of each of these metabolites in the patients presenting with SMI compared with control patients presenting to the cardiac catheterization suite with nonacute cardiovascular disease (Figure 3A). There was concordance of both the direction and magnitude of differences in the SMI cohort with changes in the PMI cohort. We generated a simple composite score by summing the equally weighted intensities for each of these metabolites and confirmed that absolute MS intensity units in addition to relative changes successfully distinguished SMI patients from controls $(P=0.004$; Figure $3 \mathrm{~B})$. This revealed excellent discriminatory ability, with receiver-operating-characteristic area under the curve (AUC) of 0.88 (Figure 3C). The composite score further confirmed that metabolic biomarkers derived in the PMI model were similarly altered in the SMI samples.
Validation of metabolic changes using isotope standards. Suppression or enhancement of the MS signal can occur from constituents present in a complex biological matrix such as plasma. We therefore performed additional studies using isotope-labeled metabolite standards for key metabolites in Tables 2-7 to provide unambiguous identification and quantification (see Methods). Supplemental Table 2 (supplemental material available online with this article; doi:10.1172/JCI35111DS1) shows absolute and relative concentration changes for the metabolites with available isotope standards, relevant to Tables 2 and 3 . The magnitude of changes in the original and follow-up studies were highly correlated $\left(r^{2}=0.9, P=0.01\right)$. Similarly, the results in Supplemental Table 3 compare peripheral and coronary sinus samples using the isotopically labeled standards, relevant to Tables 6 and 7. These separate analyses also underscored our initial findings; the magnitude of changes in the original and followup studies were again highly correlated $\left(r^{2}=0.87, P=0.01\right)$. Data for malic acid and glutamic acid, 2 metabolites that change early in the coronary sinus, are provided in Supplemental Table 4. Isotope standards were available for 3 of the 4 metabolites in the multimarker panel: TMNO, threonine, and hypoxanthine. Repeat analyses incorporating the isotope standards for these 3 markers in the PMI sub- 
Table 4

Relative changes in plasma metabolite levels in coronary sinus versus periphery 10 minutes after PMI

\begin{tabular}{|c|c|c|c|c|c|c|}
\hline \multirow[t]{2}{*}{ Metabolite } & \multicolumn{2}{|c|}{ Coronary sinus } & \multicolumn{2}{|c|}{ Periphery } & \multicolumn{2}{|c|}{ Coronary sinus vs. periphery } \\
\hline & Change & $P^{A}$ & Change & $P A$ & $P$ & Ratio \\
\hline 1-Methylhistamine & $11.2(-1.5$ to 30.5$)$ & 0.049 & $-3.5(-6.0$ to 16.7$)$ & 0.39 & 0.25 & $N A^{B}$ \\
\hline Aminoisobutyric acid & $1.6(-18.1$ to 36.1$)$ & 0.27 & $15.1(6.8$ to 48.1$)$ & 0.01 & 0.11 & 0.1 \\
\hline Hypoxanthine & 237.9 (46.2 to 313.7 ) & 0.011 & $128.1(6.8$ to 261.1$)$ & 0.010 & 0.024 & 1.9 \\
\hline Inosine & 86.9 (9.3 to 220.7) & 0.019 & $10.6(-18.7$ to 87.5$)$ & 0.091 & 0.013 & 8.2 \\
\hline Isoleucine/leucine & $6.0(-0.6$ to 13$)$ & 0.08 & $8.3(5.1$ to17.0) & 0.0006 & 0.82 & 0.7 \\
\hline Malonic acid & 32.8 (15.8 to 112.8 ) & 0.0035 & 15.0 (9.7 to 69.2$)$ & 0.026 & 0.17 & 2.2 \\
\hline Xanthine & $31.9(-19.1$ to 52.5$)$ & 0.32 & $20.7(13.1$ to 37.6$)$ & 0.04 & 0.89 & 1.5 \\
\hline
\end{tabular}

Shown are metabolites that increased in the periphery at 10 or 60 minutes after PMI, listed in alphabetical order. Change values denote median percent change versus baseline; values in parentheses represent interquartile range. ${ }^{A}$ Versus baseline. ${ }^{B}$ Discordant direction of change precluded calculation of a ratio.

jects versus cardiac catheterization controls (Supplemental Figure 3) were highly consistent with our initial findings (Figure 3A).

Functional consequences of metabolic changes. We used an in vitro cardiomyocyte model to investigate whether metabolites altered in PMI modulate the response to hypoxic challenge. Baseline apoptosis of cultured rat cardiomyocytes was $12.0 \% \pm 1.9 \%$, which increased to $24.2 \% \pm 2.2 \%(P=0.0001)$ after 3 hours of hypoxic challenge (see Methods) as assessed by FITC-conjugated annexin V staining, consistent with prior studies (20). Cell growth media was supplemented with candidate coronary sinus-enriched metabolites at the time of onset of hypoxia at concentrations approximately 2 - to 10 -fold those previously reported in the plasma (21-25). Figure $4 \mathrm{~A}$ shows the percent change in apoptosis relative to the hypoxia control for the lowest concentration of each metabolite screened. Consistent with prior studies, pretreatment of cardiomyocytes with inosine diminished apoptosis (26), while hypoxanthine potentiated apoptosis following hypoxia (27). Xanthine, glycerol-3-phosphate, and malonic acid also diminished the hypoxia-triggered apoptotic response. Figure 4B shows a dose-dependent inhibition of apoptosis at physiologically relevant concentrations of inosine more than 100 -fold lower than previously reported (26).

\section{Discussion}

Although emerging metabolomics profiling technologies hold enormous promise for illuminating biology, applications to human disease are still lacking. We therefore chose a clinical model of MI that allows for precise kinetic analysis in patients who served as their own controls. This experimental design markedly enhanced our power to identify statistically meaningful changes, while the targeted MS platform, in which metabolites are analyzed with high sensitivity and structural specificity, allowed us to unambiguously identify metabolites and thus avoid potentially confounding clinical variables (7). We hypothesized that small biochemicals may leak from injured myocardial cells before cellular damage would permit egress of macromolecules, potentially allowing for earlier detection of disease (28). Presently used indicators of myocardial injury (i.e., the myocardial isoform of CK-MB and cardiac troponins) reflect leakage of cardiac enzymes or structural proteins from irreversibly damaged cardiomyocytes, and are not reliably detected until at least 4 hours after myocardial injury (16). In contrast, the metabolic changes we identified in the PMI derivation cohort were readily apparent as early as 10 minutes after injury and were serially validated in distinct clinical cohorts. Coronary sinus catheterization provided the opportunity to sample directly from the organ of interest and served as another layer of validation that underscored the biological plausibility of the observed association between metabolic changes and MI. Finally, metabolite changes observed in humans modulated the response to hypoxic injury in vitro, which suggests that these metabolites not only serve as markers, but may also modulate the evolving response to ischemia in vivo.

\section{Table 5}

Additional metabolites enriched in the coronary sinus 10 minutes after PMI

\begin{tabular}{|c|c|c|c|c|c|c|}
\hline \multirow[t]{2}{*}{ Metabolite } & \multicolumn{2}{|c|}{ Coronary sinus } & \multicolumn{2}{|l|}{ Periphery } & \multicolumn{2}{|c|}{ Coronary sinus vs. periphery } \\
\hline & Change & $P^{A}$ & Change & $P^{A}$ & $P$ & Ratio \\
\hline Taurine & 15.1 (11.8 to 19.6$)$ & 0.003 & $-2.0(-6.3$ to 4.3$)$ & 0.60 & 0.0084 & $N A^{B}$ \\
\hline $\begin{array}{l}\text { Ribose-5-phosphate/ } \\
\text { ribulose-5-phosphate }\end{array}$ & $18.1(2.70$ to 31.0$)$ & 0.011 & $-7.3(-11.5$ to 25.3$)$ & 0.65 & 0.057 & $N A^{B}$ \\
\hline DCMP & 32.9 (17.3 to 77.4$)$ & 0.0499 & $-1.7(-28.1$ to 10.6$)$ & 0.95 & 0.036 & $N A^{B}$ \\
\hline Lactic acid & 23.5 (7.3 to 40.7$)$ & 0.021 & $0.06(-3.8$ to 8.4$)$ & 0.97 & 0.010 & 394.5 \\
\hline AMP & $102.7(16.8$ to 161.4$)$ & 0.0499 & $10.1(-7.5$ to 23.1$)$ & 0.39 & 0.023 & 10.2 \\
\hline Malic acid & 29.9 (19.5 to 42.2) & 0.003 & $6.9(-1.2$ to 29.6$)$ & 0.12 & 0.057 & 4.3 \\
\hline Glutamine & 9.7 (6.9 to 12.6) & 0.003 & $5.8(-1.1$ to 9.3$)$ & 0.26 & 0.011 & 1.7 \\
\hline Glutamic acid & 55.4 (22.0 to 68.5$)$ & 0.002 & 37.6 (18.8 to 49.2$)$ & $<0.0001$ & 0.060 & 1.5 \\
\hline
\end{tabular}


Table 6

Relative changes in plasma metabolite levels in coronary sinus versus periphery 60 minutes after PMI

\begin{tabular}{lcccccc} 
Metabolite & \multicolumn{2}{c}{ Coronary sinus } & \multicolumn{2}{c}{ Periphery } & \multicolumn{2}{c}{ Coronary sinus vs. periphery } \\
& \multicolumn{1}{c}{ Change } & $\boldsymbol{P A}$ & Change & $\boldsymbol{P A}$ & $\boldsymbol{P}$ & Ratio \\
1-Methylhistamine & $17.0(9.7$ to 33.0$)$ & 0.006 & $12.1(4.7$ to 30.7$)$ & 0.05 & 0.19 & 1.4 \\
Aminoisobutyric acid & $46.8(32.1$ to 91.2$)$ & 0.0001 & $45.6(21.4$ to 93.3$)$ & 0.003 & 0.95 & 1.01 \\
Hypoxanthine & $331.6(45.3$ to 560.7$)$ & 0.0009 & $275.1(104.3$ to 407.2$)$ & 0.0003 & 0.041 & 0.66 \\
Inosine & $75.3(-0.7$ to 124$)$ & 0.02 & $57.6(-1.9$ to 198.4$)$ & 0.03 & 0.078 & 1.3 \\
Isoleucine/leucine & $13.8(0.5$ to 18.3$)$ & 0.01 & $9.8(5.2$ to 17.6$)$ & 0.003 & 0.88 & 0.4 \\
Malonic acid & $27.0(17.7$ to 36.4$)$ & 0.008 & $33.8(12.5$ to 91.4$)$ & 0.002 & 0.28 & 0.53 \\
Xanthine & $34.3(18.7$ to 59.4$)$ & 0.0009 & $64.7(40.2$ to 88.0$)$ & 0.01 &
\end{tabular}

Shown are metabolites that increased in the periphery at 10 or 60 minutes after PMI, listed in alphabetical order. Change values denote median percent change versus baseline; values in parentheses represent interquartile range. AVersus baseline.

The metabolic, structural, and functional consequences of ischemia and/or reperfusion have been examined in a wide variety of experimental animal models, including regional ischemia after coronary vessel occlusion in dogs (29-31) and swine (32-35) as well as in humans with coronary disease (36-40) or undergoing heart surgery (41-43). However, prior studies have generally assayed relatively limited subsets of metabolites in focused approaches. Some prior studies have also relied on invasive instrumentation, such as the implantation of dialysate catheters into the myocardium (32). In support of the applicability of a targeted liquid chromatography-MS (LC-MS) platform to human cardiovascular disease, we confirmed changes in metabolites that had been previously reported as appearing in either plasma or effluent from isolated, perfused hearts (32). These changes included the sequential purine degradation products (ADP, AMP, inosine, hypoxanthine, and xanthine), intermediates of anaerobic glycolysis (lactic acid) and the TCA cycle (succinic acid), and amino acids known to be concentrated within cardiomyocytes (taurine and glutamic acid) (32).

Analysis of patient plasma using a comprehensive LC-MS platform also identified additional metabolites in these pathways that had not to our knowledge been previously reported, such as the TCA cycle intermediates malic acid and aconitic acid. We have also identified what we believe to be novel changes in human plasma levels of pyrimidine metabolites, including orotic acid, malonic acid, and aminoisobutyric acid. Alterations in TMNO, in contrast, suggest injury-mediated modulation of dietary compounds in the plasma.
The platform also identified the stable circulating derivative of the proinflammatory metabolite of histamine, methylhistamine (44).

While the application of metabolomics directly to humans has important advantages, functional studies must be pursued in complementary model systems. Our in vitro studies identified roles for several of the metabolites discussed above, including roles for glycerol-3-phosphate and malonic acid in diminishing hypoxia-triggered apoptosis in cardiomyocytes. Future studies will be necessary to compare metabolite profiles in intracellular versus extracellular compartments, to define tissue-specific responses to ischemia and injury, and ultimately to further define the functional roles for the observed metabolic alterations in animal models.

From a clinical perspective, metabolic responses arising uniquely from an abnormal hypertrophic myocardial substrate or the direct effects of ethanol must be considered; these could potentially restrict broader application to general populations. Ultimately, the validation of our findings in SMI patients mitigates the concern that ethanol-induced thrombosis and downstream injury generates a different metabolic profile from infarction as a result of coronary plaque rupture. However, further testing in larger cohorts will be necessary to confirm these preliminary findings, to define the specificity of the metabolic changes in the presence of comorbid conditions, and to extend their relevance to subgroups based on sex and race. Furthermore, while the findings in the PMI cohort suggest that our early markers are particularly useful in diagnosing very recent onset of injury, cohorts of "normal" MI patients presenting soon after symptom onset will be needed to

Table 7

Additional metabolites enriched in the coronary sinus 60 minutes after PMI

\begin{tabular}{lcc} 
Metabolite & \multicolumn{2}{c}{ Coronary sinus } \\
& \multicolumn{1}{c}{ Change } & PA \\
Glycerol-3-phosphate & $31.3(1.2$ to 65.4$)$ & 0.0092 \\
Orotic acid & $22.4(8.1$ to 39.7$)$ & 0.0063 \\
Succinic acid & $14.3(-0.4$ to 25.7$)$ & 0.027 \\
Glycerate-2-phosphate & $12.7(1.7$ to 50.6$)$ & 0.016 \\
Taurine & $7.3(5.5$ to 13.1$)$ & 0.001 \\
Malic acid & $21.3(5.3$ to 35.3$)$ & 0.0007
\end{tabular}

\begin{tabular}{cc}
\multicolumn{2}{c}{ Periphery } \\
Change & $P^{A}$ \\
$-7.9(-17.8$ to 8.8$)$ & 0.87 \\
$0.87(-16.0$ to 16.5$)$ & 0.36 \\
$2.1(-9.6$ to 11.6$)$ & 0.47 \\
$3.1(-13.1$ to 13.4$)$ & 0.85 \\
$2.1(-6.4$ to 4.3$)$ & 0.60 \\
$9.4(-0.2$ to 17.1$)$ & 0.10
\end{tabular}

Coronary sinus vs. periphery

\begin{tabular}{lc}
\multicolumn{1}{c}{$\boldsymbol{P}$} & Ratio \\
0.0063 & NAB $^{B}$ \\
0.01 & 25.9 \\
0.043 & 6.9 \\
0.042 & 4.0 \\
0.041 & 3.4 \\
0.002 & 2.3
\end{tabular}



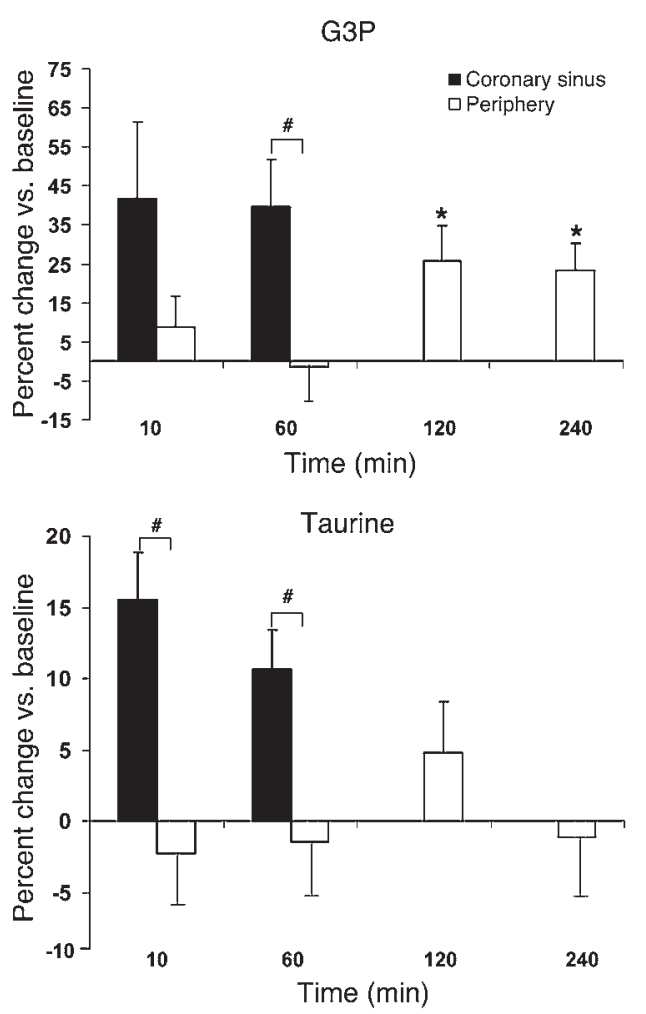

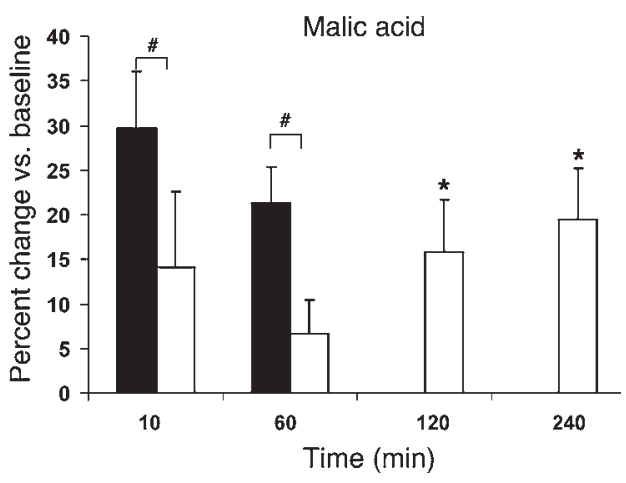

Glutamic acid

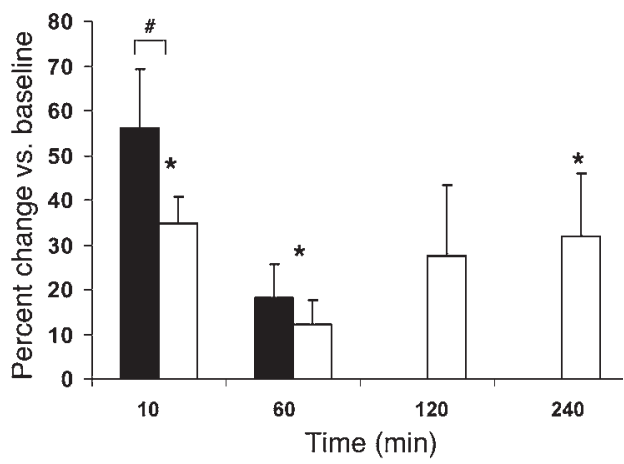

\section{Figure 2}

Kinetic analyses of representative metabolites that are enriched in the coronary sinus after myocardial injury. Data (mean \pm SEM) are restricted to the 13 patients who had simultaneous peripheral blood and coronary sinus sampling performed. Black and white bars show changes in coronary sinus and peripheral levels, respectively. G3P, glycerol-3-phosphate. ${ }^{*} P<0.05$ vs. baseline in peripheral blood. ${ }^{~} P<0.05$ between coronary sinus and peripheral samples. further assess its clinical performance. Complementary studies of other body fluids, particularly urine, may also provide insight into metabolites that are rapidly cleared from the circulation.

It is unlikely that the metabolic changes we observed herein are attributable to medication exposures. Aspirin, beta blockers, and heparin were used almost uniformly in each of the cohorts studied (Table 1), removing them as potential confounders. When subjects were stratified by calcium channel blocker or statin use, no significant differences in metabolic responses were observed for any of the metabolites in Tables $2-5$ ( $P>0.05$ for each). Large-scale studies will ultimately be necessary to identify any subtle potential metabolic alterations secondary to medications.

The recognized advantages of coupling LC with targeted MS/MS analysis include greatly increased sensitivity and highly specific identification of analytes. Targeted MS methods provide absolute measurement of analyte concentrations when appropriate standards are added, as we demonstrated in the present study. A targeted approach, however, is blind to changes in metabolites whose
A

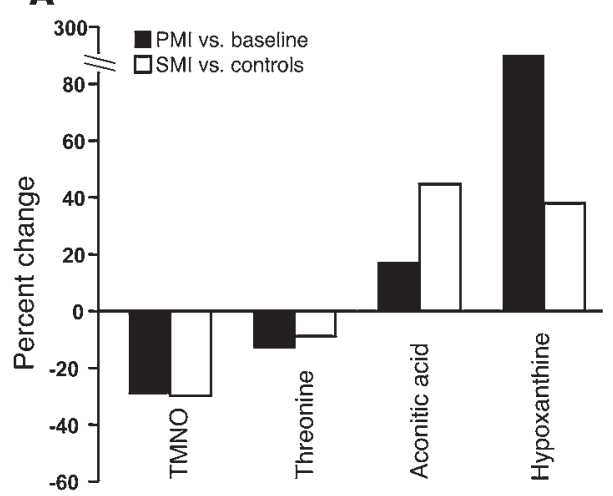

B

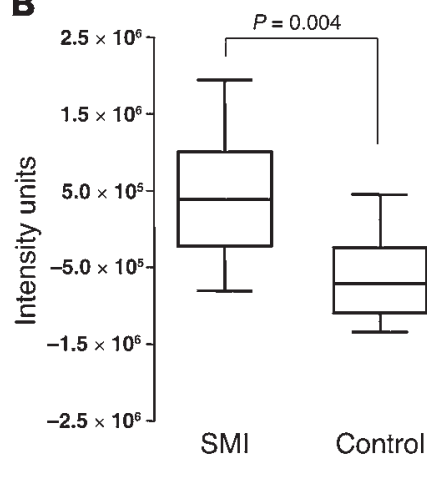

C

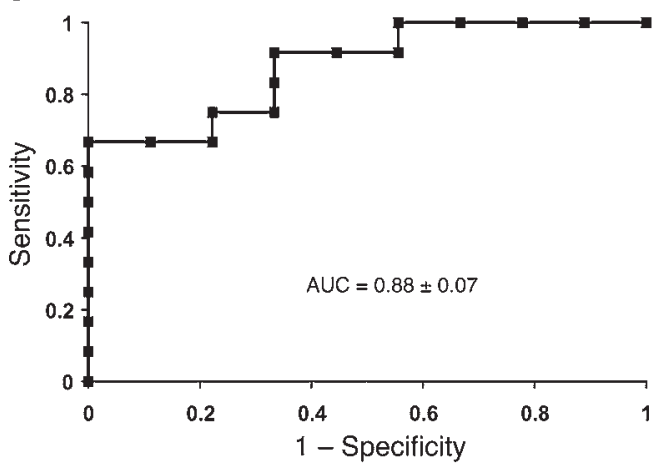

Figure 3

Metabolites whose levels significantly changed from baseline at 60, 120, and 240 minutes in PMI patients assessed in an independent SMI cohort. (A) Black bars denote average of median changes across the 3 time points compared with baseline values in PMI patients. White bars denote relative levels of each metabolite in patients presenting with SMI compared with control patients presenting to the cardiac catheterization suite with nonacute cardiovascular disease. (B) Composite scores for metabolites in A, derived by taking the weighted sum of metabolites that increased in PMI minus the sum of metabolites that decreased in PMI. Boxes denote interquartile range, lines denote median, and whiskers denote tenth and ninetieth percentiles. (C) Receiver-operating-characteristic curve for composite metabolite scores for each patient in the SMI cohort (cases) and the second control cohort (controls). AUC (mean \pm SEM) is shown. 

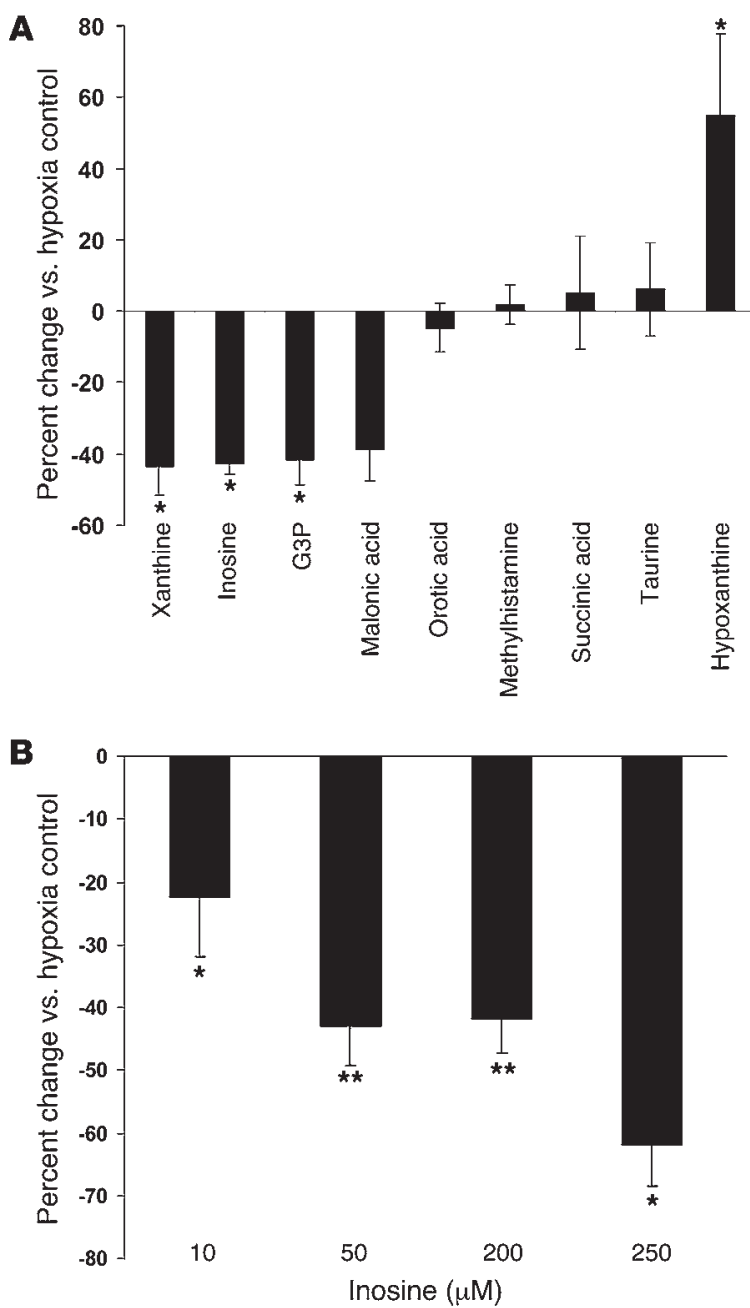

Figure 4

Cultured rat cardiomyocytes were subjected to a 3-hour hypoxic challenge as described in Methods. Cells were incubated with the indicated exogenous metabolites across a range of concentrations. Upon reoxygenation, apoptosis was assessed by FITC-labeled annexin V staining. (A) Percent change in apoptosis relative to the hypoxia control for the lowest concentration of each metabolite screened: xanthine, $14 \mu \mathrm{M}$; inosine, $10 \mu \mathrm{M}$; glycerol-3-phosphate, $1.7 \mathrm{mM}$; malonic acid, $6.24 \mu \mathrm{M}$; orotic acid, $19 \mathrm{mM}$; methylhistamine, $1.38 \mu \mathrm{M}$; succinic acid, $200 \mathrm{mM}$; taurine, $2 \mathrm{mM}$; hypoxanthine, $40 \mu \mathrm{M}$. (B) Percent change in apoptosis relative to the hypoxia control at physiologically relevant concentrations of inosine. Representative data (mean \pm SEM) from 14 total experiments are shown. ${ }^{\star} P<0.05,{ }^{* \star} P<0.01$ vs. hypoxia control.

retention times and MS characteristics have not been incorporated into the analysis method. As efforts to define the human metabolome grow (25), we anticipate increasingly comprehensive targeted platforms for biomarker and pathway discovery.

We previously reported initial observations of the application of a more limited LC-MS/MS-based platform (10), in which many of the analytes had not been unambiguously identified, to patients undergoing cardiac exercise testing. Both the present work and the prior study detected circulating purine breakdown products, including hypoxanthine, which may not always be of cardiac muscle-specific origin. However, even with deletion of hypoxanthine from the composite score, the remaining 3 metabolites (aconitic acid, TMNO, and threonine) derived from the PMI subjects have excellent discrimination of SMI cases from controls, with AUC of 0.83 . We hypothesize that in certain clinical contexts, such as myocardial ischemia at rest, elevations in hypoxanthine would be informative. In contrast, in the setting of vigorous exercise or diffuse muscle injury, the specificity of hypoxanthine for myocardial injury would be diminished. The present results also suggested the modulation of a distinct subset of TCA cycle intermediaries, amino acids, and amines not observed in the exercise cohort, which may reflect differences in the human models or distinct metabolic signatures of myocardial ischemia and MI. Ultimately, the integration of multiple data sets will be necessary for large-scale validation in patients to identify plasma biomarkers of myocardial ischemia and early injury, for which no circulating biomarkers currently exist (45).

In summary, we demonstrated the application of a metabolomics platform to a carefully phenotyped patient cohort for the discovery of blood markers with the potential to detect the presence of very early myocardial injury. We identified abnormalities in circulating metabolites as soon as 10 minutes after myocardial injury, a time frame in which, to our knowledge, no currently used biomarkers are elevated. Beyond the diagnostic utility we demonstrate here, other metabolic signatures may predict disease, establish a reference for return to normality, or aid in the design of new therapeutics for metabolic modulation.

\section{Methods}

Patients with HOCM undergoing septal ablation. A total of 36 patients undergoing PMI using alcohol septal ablation for the treatment of symptomatic HOCM were included in this study. Inclusion criteria for this cohort were (a) primary HOCM; (b) septal thickness of $16 \mathrm{~mm}$ or greater; (c) resting outflow tract gradient of greater than $30 \mathrm{mmHg}$, or an inducible outflow tract gradient of at least $50 \mathrm{mmHg}$; (d) symptoms refractory to optimal medical therapy; and (e) appropriate coronary anatomy. The most proximal accessible septal branch was instrumented using standard angioplasty guiding catheters and guidewires and 1.5 or $2.0 \mathrm{~mm} \times 9 \mathrm{~mm}$ Maverick balloon catheters. Radiographic and echocardiographic contrast injections confirmed proper selection of the septal branch and balloon catheter position. Ethanol was infused through the balloon catheter at $1 \mathrm{ml} / \mathrm{min}$. Additional injections in the same or other septal branches were administered as needed in order to cease blood flow to the isolated myocardium and reduce the gradient to less than $20 \mathrm{mmHg}$ (46).

All protocols for obtaining blood from patients, including the coronary sinus sampling protocol, were approved by the Massachusetts General Hospital Institutional Review Board, and all subjects gave written informed consent. Blood was drawn at baseline, just prior to the onset of the ablation, and at 10 minutes and 1, 2, 4, and 24 hours after the onset of injury (Supplemental Figure 1). Of the 36 patients, 13 consented to the placement of a coronary sinus catheter during the ablation, allowing for the simultaneous sampling from coronary sinus and femoral catheters at the baseline, 10-minute, and 60-minute time points. The coronary sinus catheter was subsequently removed prior to the patient leaving the catheterization suite.

A control cohort of 16 patients undergoing elective, diagnostic cardiac catheterization for cardiovascular disease, but not acute myocardial ischemia, was also enrolled. Blood was drawn prior to the onset of cardiac catheterization and at 10 and 60 minutes after the procedure was begun.

Patients with SMI. We enrolled a cohort of 12 patients undergoing emergent cardiac catheterization for acute ST-segment elevation SMI within 8 hours of symptom onset. For this cohort, blood samples were obtained in the coronary catheterization suite. For the validation studies in SMI subjects, a second control cohort of 9 patients undergoing elective, diagnostic 
cardiac catheterization for cardiovascular disease, but not acute myocardial ischemia, was also enrolled.

Blood sample processing. Samples were obtained from femoral venous catheters during the procedure, or, where indicated, from a catheter placed in the coronary sinus. Samples were collected in $\mathrm{K}_{2}$ EDTA-treated tubes (BD). All blood samples were centrifuged at 2,000 $\mathrm{g}$ for 10 minutes to pellet cellular elements. The supernatant plasma was then aliquoted and immediately frozen at $-80^{\circ} \mathrm{C}$ to minimize freeze-thaw degradation. Additional blood samples were sent to the clinical chemistry laboratory for evaluation of the standard cardiac markers CK, CK-MB, and troponin T (Roche Diagnostics).

Metabolite selection for platform. We incorporated metabolites into our platform based on the following criteria. (a) With regard to biological diversity, we chose metabolites to provide sentinels in as many of the known metabolic pathways as possible (i.e., TCA cycle intermediates, purine biosynthesis, etc.). (b) With regard to biological relevance, we specifically added some metabolites with specific potential relevance to human metabolic and/or cardiovascular diseases. (c) With regard to biophysical characteristics, we eliminated metabolites that were not readily detectable by LC-MS methods or would require the addition of highly specific methods that could not be readily generalized to other classes of compounds. We also consulted available databases with reported concentrations to assess the feasibility of identifying specific metabolites in plasma.

HPLC and MS analysis. For the analysis of sugars, ribonucleotides, and organic acids, $200 \mu \mathrm{l}$ plasma was subjected to ethanol precipitation $(80 \%$ ethanol, $19.9 \% \mathrm{H}_{2} \mathrm{O}$, and $0.1 \%$ formic acid) at $4{ }^{\circ} \mathrm{C}$ for 2 hours and centrifuged at $15,000 \mathrm{~g}$ for 15 minutes, and the supernatant was evaporated in a nitrogen chamber at $30^{\circ} \mathrm{C}$ (Caliper Life Sciences). Samples were reconstituted in $60 \mu 1 \mathrm{H}_{2} \mathrm{O}$, and aliquots were separated sequentially and automatically by injection onto 3 HPLC columns with orthogonal separation characteristics as previously described (10). Sugars and ribonucleotides were separated on a Luna amino column (Phenomenex) under normal phase using acetonitrile, water, $0.25 \%$ ammonium hydroxide, and $10 \mathrm{mM}$ ammonium acetate $(\mathrm{pH} 11)$ in a run time of 3.5 minutes. Organic acids were separated using a Synergi Polar-RP column (Phenomenex) under reverse phase using acetonitrile, water, and $5 \mathrm{mM}$ ammonium acetate ( $\mathrm{pH}$ 5.6-6.0) in a run time of 3.5 minutes. For the analysis of amino acids, plasma was diluted 10-fold with $\mathrm{H}_{2} \mathrm{O}$ and then subjected to reverse phase chromatography on a Luna phenyl-hexyl column (Phenomenex) using acetonitrile, water, and $0.1 \%$ acetic acid $(\mathrm{pH} 3.5-4.0)$ in a run time of 1.5 minutes.

The 3 HPLC columns were connected in parallel via an automated switching valve on a robotic sample loader (Leap Technologies) to a triple quadrupole mass spectrometer (AB4000Q; Applied Biosystem/Sciex), operated in electrospray ionization mode using a turbo ion spray LC-MS interface. Either positive or negative ions were selected for targeted MS/ MS analysis using selective reaction monitoring conditions. A total of 210 known metabolites were monitored for each sample. Precursor and product ions of metabolites are shown in Supplemental Table 5. Metabolite quantification was performed by integrating peak areas for parent/daughter ion pairs using Multiquan software (version 1.0; Applied Biosystem/ Sciex), and subsequently, all metabolite peaks were manually reviewed for peak quality in a blinded manner prior to statistical analysis.

LC-MS analyses with isotope standards. Representative metabolites from each of the tables were purchased from Cambridge Isotope Laboratories (Supplemental Table 1). LC-MS analyses ensured purity of greater than 98\% (data not shown), and dose-response curves were established first in solvent, and subsequently in plasma. Endogenous levels of all metabolites were more than 2 orders of magnitude above the limit of quantitation and fell well within the linear range of the dose-response relationship (Supplemental Figure 2). The indicated amount of each metabolite (Supplemental Table 1) was added to plasma prior to sample preparation, and absolute quantitation was then determined by the ratio of the endogenous analyte peak to the isotope standard peak.

In vitro cardiomyocyte hypoxia model. Protocols for cardiomyocyte procurement and use in this study were approved by the IACUC of Beth Israel Deaconess Medical Center. Cardiomyocytes were isolated from 1-day-old rats and cultured for 72 hours, as previously described (20), in $100 \mathrm{~mm}$ dishes. A 3-hour hypoxic challenge was performed in a chamber (BillupsRothenberg) filled with less than $0.5 \% \mathrm{O}_{2}, 95 \%$ nitrogen, and $5 \% \mathrm{CO}_{2}$ at $37^{\circ} \mathrm{C}$ for 3 hours. Cells were incubated with exogenous metabolites across a range of concentrations. Upon reoxygenation, apoptosis was assessed by staining with FITC-labeled annexin V (Invitrogen). Counterstaining with Hoechst $33258(4 \mathrm{mg} / \mathrm{ml}, 1: 10$ dilution in PBS of $40 \mathrm{mg} / \mathrm{ml}$ stock solution in DMSO; Invitrogen) allowed for normalization of annexin-labeled cells to total cell number.

Statistics. For clinical characteristics, values for continuous variables are presented as mean $\pm \mathrm{SD}$, and comparisons between groups were performed using 2-tailed, 2-sample Student's $t$ tests. Association between categorical variables was assessed using Fisher's Exact test.

We performed preliminary studies using sample preparation and MS replicates of pooled human samples to assess the coefficient of variation (calculated as $\mathrm{SD} /$ mean of data set; expressed as a percentage) for the metabolites in the platform. This analysis showed that the aggregate coefficient of variation was approximately $20 \%$. From the 36 patients in whom peripheral samples were collected in the PMI study, 20 patients were randomly selected for analysis as a derivation set. Levels of metabolites were tested for statistically significant percent change from baseline using the Wilcoxon signed-rank test. We used a significance threshold of $P<0.005$ in the derivation cohort, because this threshold would be expected to yield approximately 1 false positive discovery of 210 metabolites analyzed, assuming independent hypotheses. False discovery rate for a given metabolite is the fraction of metabolites with the observed $P$ value or lower that correspond to the null distribution. Conservative (i.e., upper bound) estimates of false discovery rate were obtained via the Benjamini-Hochberg procedure (47). The procedure was applied to the set of 210 measured metabolites for the comparisons of 10 - and 60-minute time points with baseline.

Metabolites that changed significantly at the 10 - or 60-minute time points in the PMI derivation cohort, and did not change significantly $(P>0.01)$ between the same time points in the control cohort of patients undergoing diagnostic catheterization without MI, were selected as candidate early biomarkers for testing in the PMI validation cohort that consisted of 16 patients. Criteria for validation was $P<0.05$ by Wilcoxon signedrank test with the direction of change concordant with that observed in the derivation cohort. The relationship between change in metabolites in the derivation and validation cohorts was assessed with a Spearman correlation coefficient. Data in all tables indicate median percent change from baseline and interquartile ranges.

Further analysis was carried out in the subgroup of 13 PMI patients with matched coronary sinus and peripheral samples. Metabolites were considered to be enriched in the coronary sinus if they changed significantly in the coronary sinus at 10 or 60 minutes compared with baseline $(P<0.05$, Wilcoxon signed-rank test) and changed to a greater extent in the coronary sinus than in the periphery (median change in coronary sinus at least 1.3 -fold that in the periphery; $P<0.05$, Wilcoxon signed-rank test).

To evaluate whether metabolic changes observed in the PMI patients were generalizable to SMI, we selected all of those metabolites that displayed significant changes from baseline at 1,2, and 4 hours in the derivation and validation PMI cohorts ( $P<0.05$ at all 3 time points). A Wilcoxon rank-sum test was used to examine levels of these individual metabolites in the patients presenting with SMI compared with control patients presenting to the cardiac catheterization suite with nonacute cardiovascular dis- 
ease. These metabolites were also compiled into a composite MS intensity unit score for SMI and control patients. To ensure equal weighting of each metabolite in this composite score, the intensity values of each metabolite were rescaled to have a common median intensity of $1.0 \times 10^{6}$ arbitrary units. The composite score was defined as the sum of metabolites that increased in PMI minus the sum of metabolites that decreased in PMI.

\section{Acknowledgments}

The authors gratefully acknowledge support from the NIH (grants R01 HL072872 to R.E. Gerszten and M.S. Sabatine; U01HL083141 to R.E. Gerszten, M.S. Sabatine, and F.P. Roth; and R01DK081572 to R.E. Gerszten), the Donald W. Reynolds Foundation (to R.E. Gerszten and M.S. Sabatine), Fondation Leducq (to A. Rosenzweig and R.E. Gerszten), Heart Failure Society of America (to G.D. Lewis), an American Heart Association Fellow-to-Faculty Award (to G.D. Lewis), the Harvard/MIT Clinical Investigator Training Pro- gram (to G.D. Lewis), and the Broad Institute Scientific Planning and Allocation of Resources Committee to establish the metabolomics platform. A. Asnani was supported by a predoctoral award from the Sarnoff Cardiovascular Research Foundation. F.P. Roth was also supported in part by National Human Genome Research Institute, NIH, grants HG003224, HG0017115, NS054052, and HG004233 and by the Keck Foundation.

Received for publication January 22, 2008, and accepted in revised form July 9, 2008.

Address correspondence to: Robert E. Gerszten, Cardiology Division and Center for Immunology and Inflammatory Diseases, Massachusetts General Hospital, Room 8307, 149 13th Street, Charlestown, Massachusetts 02129, USA. Phone: (617) 724-8322; Fax: (617) 726-1544; E-mail: rgerszten@partners.org.
1. Nicholson, J.K., and Wilson, I.D. 2003. Opinion: understanding 'global' systems biology: metabonomics and the continuum of metabolism. Nat. Rev. Drug Discov. 2:668-676.

2. Raamsdonk, L.M., et al. 2001. A functional genomics strategy that uses metabolome data to reveal the phenotype of silent mutations. Nat. Biotechnol. 19:45-50.

3. Allen, J., et al. 2003. High-throughput classification of yeast mutants for functional genomics using metabolic footprinting. Nat. Biotechnol. 21:692-696.

4. An, J., et al. 2004. Hepatic expression of malonylCoA decarboxylase reverses muscle, liver and wholeanimal insulin resistance. Nat. Med. 10:268-274.

5. Beecher, C.W.W. 2003. Chap. 17 in The buman metabolome. Kluwer Academic Publishers. Boston, Massachusetts, USA. 311-319.

6. He, W., et al. 2004. Citric acid cycle intermediates as ligands for orphan G-protein-coupled receptors. Nature. 429:188-193.

7. Brindle, J.T., et al. 2002. Rapid and noninvasive diagnosis of the presence and severity of coronary heart disease using $1 \mathrm{H}$-NMR-based metabonomics. Nat. Med. 8:1439-1444.

8. Kirschenlohr, H.L., et al. 2006. Proton NMR analysis of plasma is a weak predictor of coronary artery disease. Nat. Med. 12:705-710.

9. Lee, M.S., and Kerns, E.H. 1999. LC/MS applications in drug development. Mass. Spectrom. Rev. 18:187-279.

10. Sabatine, M.S., et al. 2005. Metabolomic identification of novel biomarkers of myocardial ischemia. Circulation. 112:3868-3875.

11. Sigwart, U. 1995. Non-surgical myocardial reduction for hypertrophic obstructive cardiomyopathy. Lancet. 346:211-214.

12. Knight, C., et al. 1997. Nonsurgical septal reduction for hypertrophic obstructive cardiomyopathy: outcome in the first series of patients. Circulation. 95:2075-2081.

13. Lakkis, N.M., et al. 1998. Echocardiography-guided ethanol septal reduction for hypertrophic obstructive cardiomyopathy. Circulation. 98:1750-1755.

14. Lakkis, N.M., Nagueh, S.F., Dunn, J.K., Killip, D., and Spencer, W.H., 3rd. 2000. Nonsurgical septal reduction therapy for hypertrophic obstructive cardiomyopathy: one-year follow-up. J. Am. Coll. Cardiol. 36:852-855.

15. Yoerger, D.M., et al. 2006. Time course of pressure gradient response after first alcohol septal ablation for obstructive hypertrophic cardiomyopathy. Am. J. Cardiol. 97:1511-1514.

16. Zimmerman, J., et al. 1999. Diagnostic marker cooperative study for the diagnosis of myocardial infarction. Circulation. 99:1671-1677.

17. Ransohoff, D.F. 2005. Bias as a threat to the valid- ity of cancer molecular-marker research. Nat. Rev. Cancer. 5:142-149.

18. Ransohoff, D.F. 2004. Rules of evidence for cancer molecular-marker discovery and validation. Nat. Rev. Cancer. 4:309-314.

19. Roberts, R., Gowda, K.S., Ludbrook, P.A., and Sobel, B.E. 1975. Specificity of elevated serum MB creatine phosphokinase activity in the diagnosis of acute myocardial infarction. Am. J. Cardiol. 36:433-437.

20. Matsui, T., et al. 1999. Adenoviral gene transfer of activated PI 3-kinase and Akt inhibits apoptosis of hypoxic cardiomyocytes in vitro. Circulation. 100:2373-2379.

21. Albert, J.D., et al. 1986. Extremity amino acid metabolism during starvation and intravenous refeeding in humans. Am. J. Physiol. 251:E604-E610.

22. Scriver, C.R., Gregory, D.M., Sovetts, D., and Tissenbaum, G. 1985. Normal plasma free amino acid values in adults: the influence of some common physiological variables. Metabolism. 34:868-873.

23. Traut, T.W. 1994. Physiological concentrations of purines and pyrimidines. Mol. Cell. Biochem. 140:1-22.

24. Wevers, R.A., Engelke, U., and Heerschap, A. 1994. High-resolution $1 \mathrm{H}-\mathrm{NMR}$ spectroscopy of blood plasma for metabolic studies. Clin. Chem. 40:1245-1250.

25. Wishart, D.S., et al. 2007. HMDB: The Human Metabolome Database. Nucleic Acids Res. 35:D521-D526.

26. Goldhaber, S.Z., et al. 1982. Inosine: a protective agent in an organ culture model of myocardial ischemia. Circ. Res. 51:181-188.

27. Xia, Y., and Zweier, J.L. 1995. Substrate control of free radical generation from xanthine oxidase in the postischemic heart. J. Biol. Chem. 270:18797-18803.

28. Song, D., O'Regan, M.H., and Phillis, J.W. 1998. Mechanisms of amino acid release from the isolated anoxic/reperfused rat heart. Eur. J. Pharmacol. 351:313-322.

29. Dorheim, T.A., Wang, T., Mentzer, R.M., Jr., and Van Wylen, D.G. 1990. Interstitial purine metabolites during regional myocardial ischemia. J. Surg. Res. 48:491-497.

30. Delyani,J.A., and Van Wylen, D.G. 1994. Endocardial and epicardial interstitial purines and lactate during graded ischemia. Am. J. Physiol. 266:H1019-H1026.

31. Mei, D.A., Gross, G.J., and Nithipatikom, K. 1996. Simultaneous determination of adenosine, inosine, hypoxanthine, xanthine, and uric acid in microdialysis samples using microbore column high-performance liquid chromatography with a diode array detector. Anal. Biochem. 238:34-39.

32. Backstrom, T., et al. 2003. Cardiac outflow of amino acids and purines during myocardial ischemia and reperfusion. J. Appl. Physiol. 94:1122-1128.

33. Zemgulis, V., et al. 2001. Energy-related metabolites during and after induced myocardial infarction with special emphasis on the reperfusion injury after extracorporeal circulation. Acta. Physiol. Scand. 171:129-143

34. Hassel, B., Ilebekk, A., and Tonnessen, T. 1998. Cardiac accumulation of citrate during brief myocardial ischaemia and reperfusion in the pig in vivo. Acta. Physiol. Scand. 164:53-59.

35. Osterlund, B., et al. 2002. Myocardial ischemia induces coronary t-PA release in the pig. Acta. Anaesthesiol. Scand. 46:271-278.

36. Mudge, G.H., Jr., Mills, R.M., Jr., Taegtmeyer, H., Gorlin, R., and Lesch, M. 1976. Alterations of myocardial amino acid metabolism in chronic ischemic heart disease. J. Clin. Invest. 58:1185-1192.

37. Srinivasan, K.N., et al. 1998. Comparison of glycoprotein components, tryptophan, lipid peroxidation and antioxidants in borderline and severe hypertension and myocardial infarction. Clin. Chim. Acta. 275:197-203.

38. Wirleitner, B., et al. 2003. Immune activation and degradation of tryptophan in coronary heart disease. Eur. J. Clin. Invest. 33:550-554.

39. Turgan, N., et al. 1999. Urinary hypoxanthine and xanthine levels in acute coronary syndromes. Int. J. Clin. Lab. Res. 29:162-165.

40. Pisarenko, O.I., et al. 1989. Features of myocardial metabolism of some amino acids and ammonia in patients with coronary artery disease. Eur. Heart J. 10:209-217.

41. Smolenski, R.T., et al. 1993. Formation and breakdown of uridine in ischemic hearts of rats and humans. J. Mol. Cell. Cardiol. 25:67-74.

42. Kennergren, C., et al. 1999. Extracellular amino acids as markers of myocardial ischemia during cardioplegic heart arrest. Cardiology. 91:31-40.

43. Svedjeholm, R., et al. 1991. Myocardial uptake of amino acids and other substrates in relation to myocardial oxygen consumption four hours after cardiac operations. J. Thorac. Cardiovasc. Surg. 101:688-694.

44. Keyzer, J.J., Breukelman, H., Wolthers, B.G., Richardson, F.J., and de Monchy, J.G. 1985. Measurement of $\mathrm{N}$ tau-methylhistamine concentrations in plasma and urine as a parameter for histamine release during anaphylactoid reactions. Agents Actions. 16:76-79.

45. Morrow, D.A., de Lemos, J.A., Sabatine, M.S., and Antman, E.M. 2003. The search for a biomarker of cardiac ischemia. Clin. Chem. 49:537-539.

46. Baggish, A.L., et al. 2006. Pathological effects of alcohol septal ablation for hypertrophic obstructive cardiomyopathy. Heart. 92:1773-1778.

47. Benjamini, Y., and Hochberg, Y. 1995. Controlling the false discovery rate: a practical and powerful approach to multiple testing. Journal of the Royal Statistical Society, Series B. 57:289-300. 